\title{
Corticotropin-Releasing Hormone Neurons in the Paraventricular Nucleus Project to the External Zone of the Median Eminence: A Study Combining Retrograde Labeling with Immunocytochemistry
}

\author{
David E. Lennard, William A. Eckert and Istvan Merchenthaler \\ Functional Morphology Section, Laboratory of Molecular and Integrative Neuroscience, NIEHS, NIH, Research Triangle Park, North \\ Carolina 27709, USA.
}

Key words: corticotropin-releasing hormone, immunocytochemistry, retrograde labeling.

\begin{abstract}
Corticotropin-releasing hormone $(\mathrm{CRH})$ is the major regulator of the pituitary-adrenal axis. CRH-immunoreactive perikarya are widely distributed in the central nervous system; however, only those which participate directly in the regulation of adrenocorticotropin are connected to the portal circulation in the external zone of the median eminence. The present study describes the identification of these hypophysiotropic neurons using retrograde labeling and $\mathrm{CRH}$ immunocytochemistry. Fluoro-Gold was injected peripherally then, 5 days later, the animals were treated with colchicine. Twenty-four hours later the animals were sacrificed, and their brains were immunostained for $\mathrm{CRH}$ with the indirect immunofluorescence technique. The results indicate that the vast majority of the Fluoro-Gold-accumulating and $\mathrm{CRH}$-immunopositive perikarya (hypophysiotropic neurons) are located in the medial parvicellular subdivision of the paraventricular nucleus (PVN). However, not each $\mathrm{CRH}$-immunoreactive neuron contains Fluoro-Gold, i.e. a small portion of these neurons project to areas of the brain other than the median eminence. The anterior, lateral and periventricular subdivisions of the PVN also contain hypophysiotropic $\mathrm{CRH}$-immunoreactive perikarya, however, their number is much less than in the medial parvicellular subdivision. Scattered double-labeled cells are also present in the medial preoptic area and the dorsal hypothalamus, just behind the PVN. These results support previous observations that the PVN, particularly the medial parvicellular subdivision, is the predominant source of the hypophysiotropic CRH neurons.
\end{abstract}

Corticotropin-releasing hormone (CRH; also called corticotropinreleasing factor, CRF), a 41 amino-acid peptide $(1,2)$, is a member of the classic hypophysiotropic hormone family $(3,4)$. $\mathrm{CRH}$-immunoreactive ( $\mathrm{CRH}-\mathrm{i})$ neurons and nerve terminals are widely distributed, not only within the hypothalamus but, throughout the CNS (5-8), where CRH functions as a neurotransmitter or neuromodulator. Within the hypothalamus, most of the immunoreactive perikarya are located in the parvicellular subdivisions of the paraventricular nucleus (PVN) and in the preoptic area. Fewer $\mathrm{CRH}$-i perikarya are present in the dorsal hypothalamic area, the lateral hypothalamus, and the premammillary nuclei. Scattered $\mathrm{CRH}$-i perikarya are located in the magnocellular PVN and supraoptic nucleus. The hypophysiotropic $\mathrm{CRH}$, after being synthesized by perikarya in the hypothalamus, is transported via rapid axonal transport to the median eminence (ME) where it is stored, and upon stimulation it is released from nerve terminals of the ME into the hypophysial portal circulation. Through this circulatory system $\mathrm{CRH}$ reaches the anterior pituitary where it stimulates the release of adrenocorticotropin (ACTH), $\beta$-endorphin and $\alpha$-melanocyte-stimulating hormone $(\alpha-\mathrm{MSH})(2)$. $\mathrm{CRH}$ is also released into the vasculature of the organum vasculosum of the lamina terminalis (OVLT), the other circumventricular organ of the hypothalamus (the function of CRH released here is not known).

Attempts to identify the hypophysiotropic CRH neurons, i.e. those which form nerve terminals on capillaries of the hypophysial portal circulatory system, and thereby, directly regulate ACTH, $\beta$-endorphin and $\alpha$-MSH secretion have included: 1) implantation of anterior pituitary fragments into different brain areas; 2) ablation or stimulation of selected brain regions and measurement of the subsequent changes in the concentration of ACTH and glucocorticoids in the peripheral blood; 3) surgical or electrical lesions of different brain areas containing CRH-i neurons followed by immunocytochemical detection of $\mathrm{CRH}$ in the ME; 4) use of hypophysectomized or adrenalectomized animals in which the negative feedback effect of ACTH and/or glucocorticoids has been disrupted; and 5) electron microscopic identification of degenerating $\mathrm{CRH}-\mathrm{i}$ fibers and terminals in the $\mathrm{ME}$ following

Correspondence to: I. Merchenthaler, MD C4-07, LMIN, NIEHS, NIH, Research Triangle Park, North Carolina 27709, USA. 
lesion of their perikarya in the PVN (see review in 9). The results of these studies strongly suggested that the PVN is the major source of hypophysiotropic CRH neurons. However, because of their inherent limitations (e.g. their inability to discriminate between the location of hypophysiotropic neurons and fibers of passage and to distinguish between hypophysiotropic and nonhypophysiotropic $\mathrm{CRH}-\mathrm{i}$ neurons), most of these techniques could not provide an unequivocal identification of the neuronal population that projects to the ME. Recently, a combinative effort of retrograde labeling from the $\mathrm{ME}$ and immunocytochemical detection of endogenous $\mathrm{CRH}$ has also been introduced $(10,11)$. The present study was designed to clarify these observations by identifying the $\mathrm{CRH}$ neurons that project to the ME and the OVLT with a sensitive double labeling technique. Fluoro-Gold (FG), a retrograde tracer which does not penetrate the bloodbrain barrier, was administered peripherally and those retrogradely labeled perikarya that also contained CRH were identified by fluorescence immunocytochemistry in the same tissue section.

\section{Results}

\section{Location of FG-accumulating neurons}

Within the hypothalamus, the majority of FG-containing neurons were concentrated in well defined nuclei, such as the PVN, the anterior periventricular, the supraoptic, arcuate and periventricular preoptic nuclei, the medial preoptic area, and the diagonal band of Broca. Scattered FG-accumulating neurons were seen in the lateral hypothalamus, the accessory magnocellular nuclei and the posterior hypothalamic area. Within the PVN, the majority of the retrogradely labeled cells were located in the medial parvicellular subdivision. Somewhat less was seen in the anterior parvicellular subdivision. The lateral parvicellular subdivision contained only scattered FG-accumulating cells. In the arcuate nucleus, the retrogradely labeled perikarya occupied the dorsomedial and ventrolateral subdivisions. The ventromedial subnucleus contained only a few FG-concentrating cells. FG-containing perikarya in the diagonal band of Broca and the lateral portion of the medial preoptic area showed a tent-like arrangement, similar to those immunoreactive for luteinizing hormone-releasing hormone (10). Another group of retrogradely labeled perikarya in the preoptic region occupied the periventricular preoptic nucleus. FG-accumulating cells in the anterior periventricular nucleus were densely packed and located close to the wall of the third ventricle (see refs. 12 and 13 for details).

\section{Location of $\mathrm{CRH}$-i neurons}

In the hypothalamus, most of the CRH-i perikarya were concentrated in the parvicellular subnuclei of the PVN (14) (Fig. 1A, 1B), the medial preoptic area, the dorsal and posterior hypothalamic areas, the lateral hypothalamus, and the premammillary nuclei (see ref. 8 for details). Within the PVN, the majority of the CRH-i perikarya (an average of 140 immunopositive cells in a $30 \mu \mathrm{m}$ vibratome section) was present in the medial parvicellular subdivision. The anterior parvicellular subdivision contained less (an average of 30 immunoreactive perikarya per section) $\mathrm{CRH}-\mathrm{i}$ cells. The periventricular and lateral parvicellular subdivision contained scattered cells (an average of 10 cells per section). The dorsal cap of the PVN contained only a few CRH-i perikarya. Within the hypothalamus, two circumventricular organs contained CRH-i nerve terminals: the ME and the OVLT.

\section{Distribution of $C R H-i$ and FG-accumulating perikarya: the hypophysiotropic $C R H$-i neuronal system}

The vast majority of CRH-i perikarya which retrogradely accumulated FG were seen in the parvicellular subdivisions of the PVN (Figs. 1C, 1D and 2A-2D). Only a few double-labeled cell bodies were observed in the medial preoptic and dorsal hypothalamic areas. Interestingly, not all $\mathrm{CRH}-\mathrm{i}$ perikarya contained $\mathrm{FG}$, and not every FG-accumulating cell in these regions was immunoreactive for CRH. Within the PVN, the vast majority of the retrogradely labeled CRH-i perikarya were concentrated in the medial parvicellular subdivision. An average of $140 \mathrm{CRH}-\mathrm{i}$ perikarya were counted here and $70 \%$ of them contained FG. In the anterior and lateral parvicellular subdivisions, approximately $25 \%$ of CRH-i perikarya were retrogradely labeled. The horizontally located, large CRH-i cells above the medial parvicellular PVN and between the lateral edge of the PVN and the fornix did not concentrate FG. The distribution of $\mathrm{CRH}$-i perikarya with access to fenestrated capillaries of the ME and OVLT is represented in Table 1 and Figs. 3 and 4.

\section{Discussion}

The present study describes the topographical location of CRH-i neurons projecting to the external zone of the ME and OVLT. Since only the $\mathrm{CRH}-\mathrm{i}$ neurons that have access to fenestrated capillaries of the ME form the final common pathway regulating the function of the anterior pituitary, the identification of these hypophysiotropic neurons is of great importance. The hypophysiotropic neurons, including the $\mathrm{CRH}-\mathrm{i}$ neurons, even within a

TABLE 1. Relative Participation of Fluoro-Gold (FG)-Labeled CRH-i Perikarya in the Rat Brain.

\begin{tabular}{|c|c|c|c|c|c|}
\hline \multicolumn{3}{|c|}{ Vibratome sections } & \multicolumn{3}{|c|}{ Paraffin sections } \\
\hline CRH-i cells & FG-CRH-i cells & $\%$ & CRH-i cells & FG-CRH-i cells & $\%$ \\
\hline $736(3)$ & 444 & 60.3 & $282(ठ)$ & 209 & 74.1 \\
\hline $810(3)$ & 554 & 68.4 & $240(3)$ & 187 & 77.9 \\
\hline $642(9)$ & 424 & 66.1 & $308(9)$ & 212 & 68.8 \\
\hline $882(q)$ & 573 & 64.9 & $346(q)$ & 259 & 74.9 \\
\hline \multicolumn{6}{|l|}{ Total } \\
\hline 3070 & 1995 & $64.9 \pm 3.4$ & 1176 & 867 & $73.7 \pm 3.8$ \\
\hline
\end{tabular}



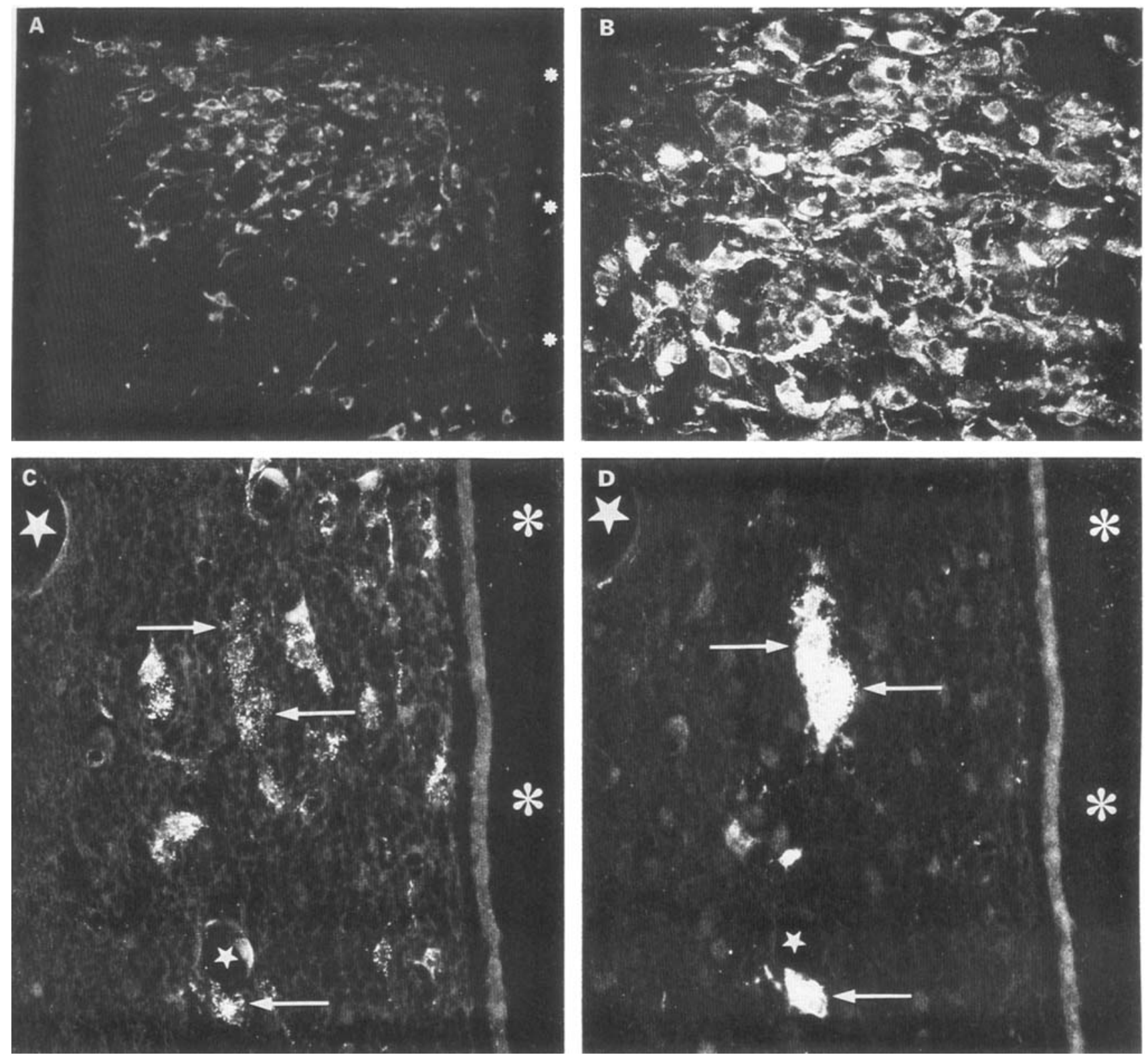

Fig. 1. CRH-i perikarya (indicated by Texas red) in a $30 \mu \mathrm{m}$ vibratome section from the medial parvicellular subdivision of the PVN (A and B). The third ventricle is represented by asterisks. CRH-i (C) and Fluoro-Gold (FG)-accumulating (D) perikarya in the periventricular subdivision of the PVN in a thin paraffin section. Those CRH-i perikarya that accumulate FG are indicated by arrows. The stars show identical blood vessels in (C) and (D). Note that each of the three CRH-i perikarya in (D) contains the retrograde tracer FG as shown in (C), but only three of the FG-containing perikarya in (C) are immunoreactive for CRH as shown in (B). (A) $\times 90$; (B) $\times 200$; (C and D) $\times 360$.

well circumscribed area or nucleus, are intermixed with nonhypophysiotropic neurons which also contain $\mathrm{CRH}$ but do not project to the ME. These neurons probably contact other neurons in the CNS where CRH functions as a neuromodulator or neurotransmitter. Our observations on the location of the hypophysiotropic CRH-i neurons are consistent with those obtained from studies using electrolytic lesion of the PVN $(15,16)$, surgical isolation of the medial basal hypothalamus (17-19), or adrenalectomy or hypophysectomy (20) followed by immunocytochemical detection of $\mathrm{CRH}$ in the ME. The results of these studies strongly suggested that the PVN was the major source of those CRH-i neurons that send their processes to the portal circulatory system.

Recently, these observations have been further supported by studies using retrograde labeling from the $\mathrm{ME}$ in combination with CRH immunocytochemistry. In one of these recent studies, the lectin wheat germ agglutinin (WGA) was injected into the surgically isolated ME (10). The retrogradely labeled perikarya were visualized with the adjacent sections method by detecting the retrograde tracer and $\mathrm{CRH}$ in two adjacent sections. Identical cell bodies immunoreactive for WGA and CRH represented the hypophysiotropic CRH-i neurons (10). Although local injection 

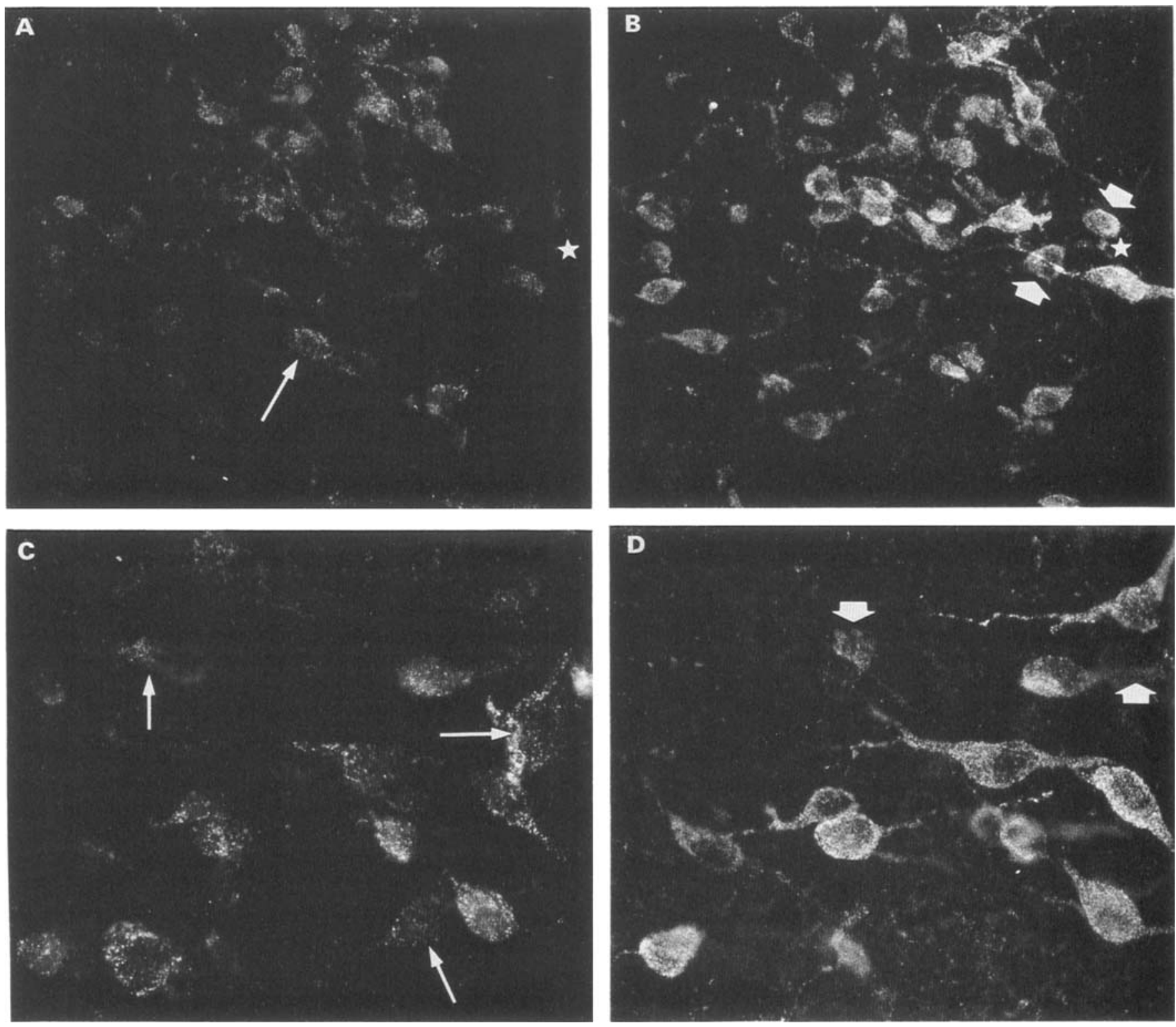

FlG. 2. CRH-i perikarya ( $\mathrm{B}$ and $\mathrm{D}$ ) and Fluoro-Gold (FG)-containing perikarya (A and $\mathrm{C}$ ) in two vibratome sections taken from two different levels of the medial parvicellular subdivisions of the PVN. Arrows in (A and (C) indicate FG-accumulating perikarya that are not immunoreactive for CRH, i.e. these cannot be seen in (B) and (D). Arrows in (B) and (D) indicate CRH-i perikarya that are not retrogradely labeled with FG, i.e. these cannot be seen in (A) and (C). Note that with the exception of a few perikarya, almost each CRH-i cell body in the medial parvicellular portion of the PVN contains FG. Stars in (A) and (B) show the same blood vessel. (A and B) $\times 200$; (C and D) $\times 360$.

of the retrograde tracer into the surgically isolated ME results in the most precisely controlled technique of administration to identify neurons that project to the ME, the technique is laborious and the amount of retrograde tracer necessary for optimal labeling cannot be precisely specified. In addition, when this technique is combined with the adjacent section technique (10), many of the double-labeled perikarya are not counted due to the difficulties in identifying the same neurons in two adjacent sections. The advantage of the adjacent section technique, however, is that it provides data without cross-reactivity problems (21).

Previously, hypophysiotropic CRH-i neurons have been identified by using True Blue, a fluorescent retrograde tracer, injected directly into the ME through the third ventricle (11). The retrogradely labeled $\mathrm{CRH}-\mathrm{i}$ cells were identified in the same cryostat sections. Although the efficiency of this technique is superior to the adjacent section technique used by Kawano et al. (10), the precise location of the ME injection site could not be adequately controlled. In addition, since the $\mathrm{ME}$ is not a densely packed structure (rather, it is spongy) spread of the tracer into areas adjacent to the $\mathrm{ME}$, including the arcuate nucleus, could not be ruled out (for technical details see ref. 12).

A good estimation of the number of retrogradely labeled neurons can be achieved by the technique used in the present study. The use of FG as a retrograde tracer (22) is very convenient, 
the supramaximal dose ensures optimal labeling, and its detection can be combined with peptide immunocytochemistry in the same tissue section. The only disadvantage of using FG to identify the hypophysiotropic CRH-i perikarya is that it is also taken up from capillaries supplying the OVLT. However, the number of nerve terminals in the external zone of the $\mathrm{ME}$ is vastly greater than the number in the OVLT and, as we have shown in these studies, the number of FG-labeled $\mathrm{CRH}$-i perikarya outside the PVN is low. These observations indicate that the primary source of CRH-i nerve terminals in the OVLT is the PVN. The findings that when the anterograde tracer, Phaseolus vulgaris, is injected into the medial parvicellular subdivision of the PVN, nerve terminals in the OVLT are labeled (23), indicate that some of the parvicellular neurons in the PVN, beside the ME, also project to

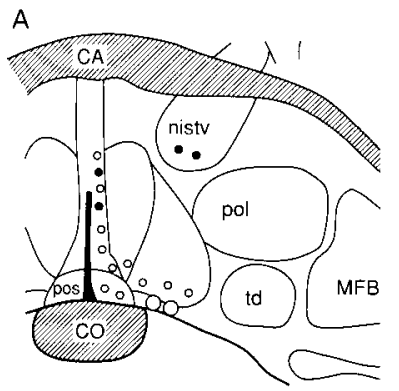

B
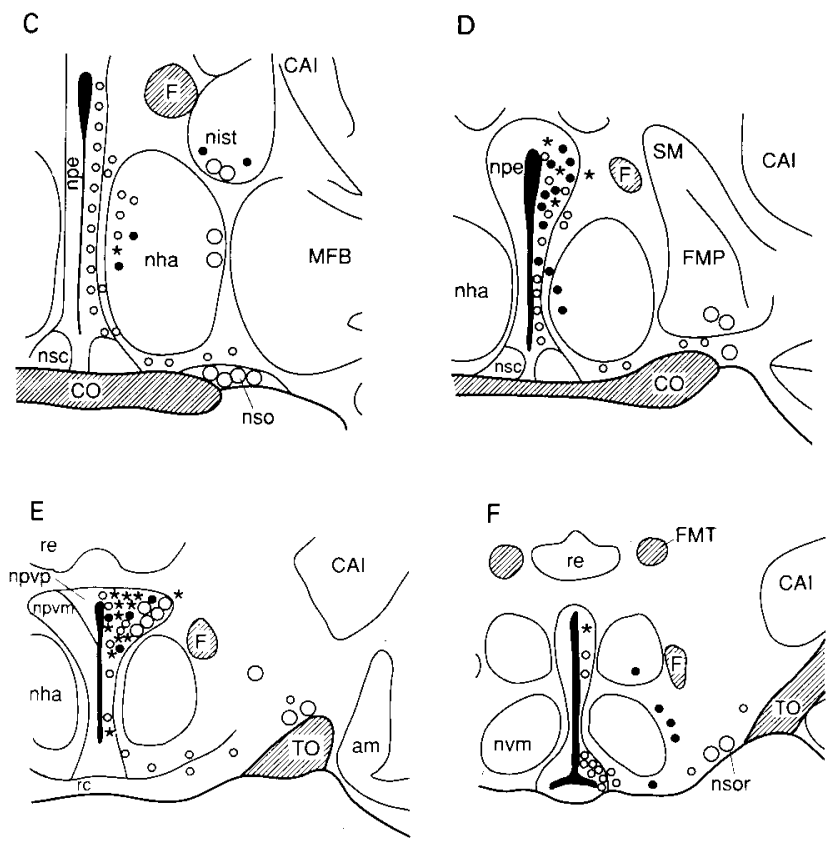

G

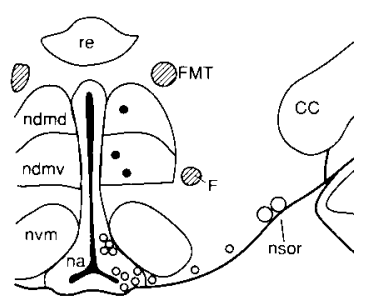

capillaries of the OVLT. FG labeling provides a stable signal in retrogradely labeled neurons in both thin paraffin and thicker, vibratome sections. By simply changing appropriate filter combinations of the microscope, both FG (metal gray fluorescence), and CRH-i (a fluorescent dye with a color different from that of FG, e.g. Texas red) can be detected. Although the detection of FG and CRH in thin paraffin sections may be less sensitive than in vibratome sections, the thin 5 to $7 \mu \mathrm{m}$ sections provide excellent spatial resolution and, therefore, superior quantitative evaluation. The amount of FG in tangentially-sectioned perikarya may be below the detection limit and, therefore, the number of hypophysiotropic neurons can be underestimated. In thicker $(30 \mu \mathrm{m})$ vibratome sections overlapping of double-labeled cells cannot be ruled out; therefore, cell counting cannot be as precise as in thin paraffin sections (compare Figs. 1C, 1D with 2A-D). The thicker vibratome sections on the other hand, provide stronger signals both for FG and the endogenous $\mathrm{CRH}$ (compare Figs. $2 \mathrm{~A}-\mathrm{D}$ with 1C, 1D). However, when densely packed nuclei are examined, such as the PVN, single- and double-labeled cells can overlap and be misinterpreted (for details see reference 10).

The results of both techniques discussed above are in good agreement with our observations and indicate that the PVN is the predominate source of the hypophysiotropic $\mathrm{CRH}-\mathrm{i}$ neurons. The few double-labeled cells seen in the posterior-dorsal hypothalamus probably also project to the ME, and the few in the preoptic area, to the OVLT (see below). In the medial parvicellular subdivision of the PVN approximately $70 \%$ of the CRH-i neurons contain FG. The number of these hypophysiotropic neurons is less (approximately 25\%) in the other subnuclei of the PVN. Interestingly, the periventricular subdivision of the PVN, which is known to project to the external zone of the $\mathrm{ME}(13,24,25)$ contained scattered CRH-i perikarya and only a few of them accumulated FG. The identification of the hypophysiotropic neurons is of great importance for understanding the regulation of these neurons, which form the final common pathway for the regulation of the anterior pituitary. FG labeling provides an

FIG. 3. Schematic representation of CRH-i (dark circles), Fluoro-Gold (FG)-accumulating (small clear circles for the parvicellular and large clear circles for the magnocellular neurosecretory system), and double-labeled (asterisks) perikarya in a series of cross sections through the rat hypothalamus. Note that the vast majority of the hypophysiotropic CRH-i cell bodies, i.e. those that accumulate the retrograde tracer FG, are located in the parvicellular subdivisions of the PVN. However, scattered hypophysiotropic cells can be found in the medial preoptic area and the dorsal hypothalamus, just behind the PVN. am-nucleus amygdaloideus medialis; $\mathrm{CA}$ - commissura anterior; $\mathrm{CAI}$ - capsula interna; $\mathrm{CC}$-crus cerebri; $\mathrm{cm}$ - nucleus magnocellularis caudalis; $\mathrm{CO}$-chiasma opticum; F-fornix; FMT - fasciculus mammillothalamicus; gp-globus pallidus; LM - lemniscus medialis; MFB - medial forebrain bundle; na-nucleus arcuatus; ndmd-nucleus dorsomedialis, pars dorsalis; ndmv-nucleus dorsomedialis, pars ventralis; nha-nucleus hypothalamicus anterior; nhp - nucleus hypothalamicus posterior; nist - nucleus interstitialis striae terminalis; nistv-nucleus interstitialis striae terminalis, pars ventralis; npe - nucleus periventricularis; npmd - nucleus premammillaris dorsalis; npmv-nucleus premammillaris ventralis; npvm - nucleus paraventricularis, pars magnocellularis; npvp - nucleus paraventricularis, pars parvicellularis; nsc - nucleus suprachiasmaticus; nso-nucleus supraopticus; nsor-nucleus supraopticus, retrochiasmatic part; nvm-nucleus ventromedialis; pol-nucleus preopticus lateralis; pom-nucleus preopticus medialis; pos-nucleus preopticus suprachiasmaticus; re-nucleus reuniens thalami; rc-retrochiasmatic area; SM - stria medullaris; td - tractus diagonalis; TO-tractus opticus. Modified after König and Klippel (27). 
180 CRH neurons projecting to the median eminence
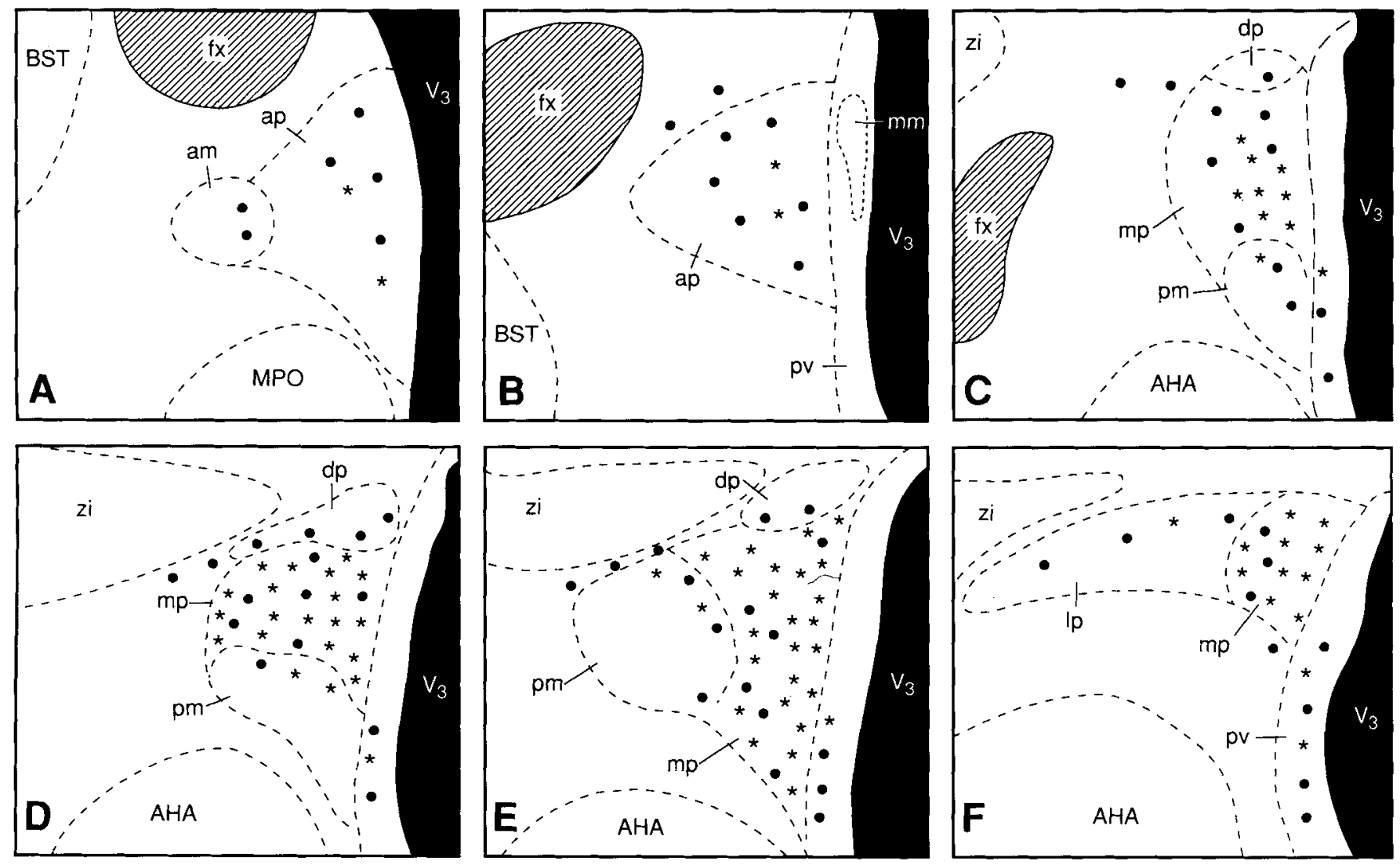

FIG. 4. Schematic representation of CRH-i perikarya (dark circles) and CRH-i perikarya that are retrogradely labeled with Fluoro-Gold (asterisks) in the PVN of the hypothalamus. Each symbol represents three CRH-i perikarya. The vast majority of the hypophysiotropic CRH-i perikarya are located in the medial parvicellular subdivision ( $\mathrm{mp}$ ) of the PVN. Less are present in the anterior parvicellular (ap), the periventricular (pv), and the lateral parvicellular (lp) subdivisions. The dorsal parvicellular subdivision (dp) contains only a few double-labeled perikarya. AHA-anterior hypothalamic area: am-anterior magnocellular subdivision of the PVN; BST - bed nucleus of the stria terminalis; fx - fornix; V3-third ventricle; pm-posterior magnocellular subdivision of the PVN. Modified after Sawchenko et al. (28).

excellent, simple, yet effective and, in most cases, specific, technique for this purpose. In the future, this type of retrograde labeling should make it possible to characterize the true hypophysiotropic neurons, i.e. their receptors, afferentations and the neuropeptides and neurotransmitters colocalized with hypophysiotropic hormones, including $\mathrm{CRH}$.

In summary, although CRH-i perikarya are widely distributed within the hypothalamus, our studies, together with previous experiments, indicate that the vast majority of the hypophysiotropic CRH-i neurons, i.e. those which directly regulate ACTH, $\beta$-endorphin and $\alpha$-MSH secretion from the anterior pituitary, is located in the medial parvicellular subdivision of the PVN. Hypophysiotropic and non-hypophysiotropic $\mathrm{CRH}-\mathrm{i}$ neurons are intermixed in the PVN, indicating that $\mathrm{CRH}$ produced even in a classical hypophysiotropic nucleus like the PVN may function not only as a hypophysiotropic factor, but as a neuromodulator or neurotransmitter.

\section{Materials and Methods}

Twelve male rats and twelve female rats (sacrificed on the day of proestrus) were injected intraperitoneally with supramaximal doses of FG $(15 \mathrm{mg} / \mathrm{kg}$ body wt dissolved in 0.9\% saline; Fluorochrome Inc.. Engelwood, $\mathrm{CO}$, USA) $(12,13,22)$. Five days later the animals were anesthetized with tribromoethanol ( $1 \mathrm{ml} / 100 \mathrm{~g}$ body wt of a $2.5 \%$ solution), and colchicine was administered into the lateral ventricle $(5 \mu \mathrm{i} / \mathrm{l} 00 \mathrm{~g}$ body wt of a $20 \mathrm{mg} /$ $\mathrm{ml}$ solution). Twenty-four hours later the animals were anesthetized again with tribromoethanol and perfused through the ascending aorta with $1 \%$ paraformaldehyde in $0.05 \mathrm{M}$ phosphate-buffered saline followed by $4 \%$ paraformaldehyde in the same buffer (10). Following overnight postfixation, five hypothalami from both sexes were embedded in paraffin and were sectioned at $8 \mu \mathrm{m}$. Seven hypothalami from both sexes were cut on a vibratome at $30 \mu \mathrm{m}$. The antiserum against $\mathrm{CRH}(\# 569)$ was raised against rat-CRH in a rabbit and was used at a dilution of 1:4,000. Biotinylated goat anti-rabbit IgG and Texas-red-labeled avidin were purchased from Jackson ImmunoResearch (West Grove. PA, USA) and were used at dilutions of $1: 500$ and 1:1,000, respectively (see ref. 10 for technical details). Following completion of the immunocytochemical procedure, the sections were dehydrated in graded alcohols and coverslipped from xylene with DePex (Fluka).

All sections were examined with an Axiophot photomicroscope equipped with the necessary excitation and emission filters for immunofluorescence microscopy. Tmax 3200 black and white and P800/1600 color films (Eastman Kodak, Co., Rochester, NY, USA) were used for photography. For quantitative analysis, two male and two female paraffinembedded and two male and two female unembedded brains were used Every tenth paraffin section and every fifth vibratome section was used for counting single-labeled ( $\mathrm{CRH}-\mathrm{i})$ and double-labeled $(\mathrm{CRH}-\mathrm{i}$ and $\mathrm{FG}$ accumulating) perikarya. By changing appropriate filter combinations single- and double-labeled CRH-i perikarya were counted. The atlas of Paxinos and Watson (26) and König and Klippel (27) were used to determine the location in the rat hypothalamus of FG-accumulating and CRH-i neurons. 


\section{Acknowledgements}

The authors wish to thank Scott Trasti for his technical help and Drs Michael D. Culler and Francisco J. Lopez, and JoAnne Reid for critical reading of the manuscript.

Accepted 21 October 1992

\section{References}

1. Rivier J, Spiess J, Vale W. (1983). Characterization of rat hypothalamic corticotropin-releasing factor. Proc Natl Acad Sci USA. 80: 485 $I-4855$.

2. Vale W, Spiess J, Rivier C. (1981). Characterization of a 4I-residue ovine hypothalamic peptide that stimulates the secretion of corticotropin and $\beta$-endorphin. Science. 213: 1394-1397.

3. Palkovits M. (1986). Neuropeptides in the median eminence. Neurochem Int. 9: 131-139.

4. Merchenthaler I. (1991). Current status of brain hypophysiotropic factors. Morphologic aspects. Trends Endocrinol Metab. 2: 219-226.

5. Olschowka JA, O'Donohue TL, Mueller GP, Jacobowitz DM. (1982). Hypothalamic and extrahypothalamic distribution of CRF-like immunoreactive neurons in the rat brain. Neuroendocrinology. 35: 305-308.

6. Bloom FE, Battenberg ELF, Rivier J, Vale W. (1982). Corticotropin releasing factor (CRF): immunoreactive neurons and fibers in rat hypothalamus. Regul Pept. 4: 43-48.

7. Bugnon C, Fellmann D, Gouget A, Cardot J. (1982). Corticoliberin in rat brain: immunocytochemical identification and localization of a novel neuroglandular system. Neurosci Lett. 30: 25-30.

8. Merchenthaler I, Vigh S, Petrusz P, Schally AV. (1982). Immunocytochemical localization of corticotropin-releasing factor (CRF) in the rat brain. Am J Anat. 165: 385-396.

9. Petrusz P, Merchenthaler I. (1992), The corticotropin-releasing factor system. In: Nemeroff CB, ed. Comprehensive textbook of neuroendocrinology. I25-179. CRC Press, Boca Raton.

10. Kawano H, Daikoku S, Shibasaki T. (1988). CRF-containing neuron systems in the rat hypothalamus: retrograde tracing and immunohistochemical studies. J Comp Neurol. 272: 260-268.

11. Niimi M, Takahara J, Hashimoto $\mathrm{K}_{1}$ Kawanishi K. (1988). Immunohistochemical identification of corticotropin releasing factor-containing neurons projecting to the stalk-median eminence of the rat. Peptides. 9: 589-593.

12. Merchenthaler I. (1990). Retrograde labeling of hypophysiotropic neurons by local injection of wheat germ agglutinin (WGA) into the median eminence or peripheral administration of Fluoro-Gold. Mol Cell Neurosci. 1: 93-106.

13. Merchenthaler I. (1991). Neurons having access to fenestrated capillaries in the central nervous system of the rat: a retrograde tracing study with Fluoro-Gold. Neuroscience. 44: 655-662.

14. Swanson LW, Kuypers HGJM. (1980). The paraventricular nucleus of the hypothalamus: cytoarchitectonic subdivisions and organization of projections to the pituitary, dorsal vagal complex, and spinal cord as demonstrated by retrograde fluorescence double-labeling method. J Comp Neurol. 194: 555-570.

15. Bruhn TO, Plotsky PM, Vale WW. (1984). Effect of paraventricular lesions on corticotropin releasing factor (CRF)-like immunoreactivity in the stalk-median eminence: studies on the adrenocorticotropin response to ether stress and exogenous CRF. Endocrinology. 114 $57-62$.

16. Liposits Zs, Lengvari I, Vigh S, Schally AV, Flerko B. (1983). Immunohistological detection of degenerating CRF-immunoreactive nerve fibers in the median eminence after lesions of paraventricular nucleus of the rat. A light and electron microscopic study. Peptides. 4: 941-953.

17. Antoni FA, Palkovits M, Makara GB, Linton EA, Lowry PJ, Kiss JZ. (1983). Immunoreactive corticotropin-releasing hormone in the hypothalamoinfundibular tract. Neuroendocrinology. 36: 415-423.

18. Merchenthaler I, Hynes MA, Vigh S, Schally AV, Petrusz P. (1984). Corticotropin-releasing factor (CRF): origin and course of afferent pathways to the median eminence (ME) of the rat hypothalamus. Neuroendocrinology. 39: 296-306.

19. Tilders FJ, Schipper HJ, Lowry PJ, Vermes 1. (1982). Effect of hypothalamus lesions on the presence of CRF-immunoreactive nerve terminals in the median eminence and on the pituitary-adrenal response to stress. Regul Pept. 5: 77-84.

20. Merchenthaler I, Vigh S, Petrusz P, Schally AV. (1983). The paraventriculo-infundibular corticotropin-releasing factor (CRF) pathway as revealed by immunocytochemistry in long-term hypophysectomized and adrenalectomized rats. Regul Pept. 5: 295-305.

21. Hökfelt T, Tsuruo Y, Meister B, Melander T, Schalling M, Everitt B (1987). Localization of neuroactive substances in the hypothalamus with special reference to coexistence of messenger molecules. Adv Exp Med Biol. 219: 21-45.

22. Schmeud LC, Fallon JH. (1986). Fluoro-Gold: a new fluorescent retrograde axonal tracer with numerous unique properties. Brain Res. 377: $147-154$

23. Larsen PJ, Moller M, Mikkelsen JD. (1991). Efferent projections from the periventricular and medial parvicellular subnuclei of the hypothalamic paraventricular nucleus to the circumventricular organs of the rat: a phaseolus vulgaris-leucoagglutinin (PHA-L) tracing study. J Comp Neurol. 307: 462-479.

24. Lechan RM, Nestler JL. Jacobson S, Reichlin S. (1980). The hypothalamic tuberoinfundibular system of the rat as demonstrated by horseradish peroxidase (HRP) microiontophoresis. Brain Res. 195: 13-27.

25. Wiegand SJ, Price JL. (1980). The cells of origin of the afferent fibers to the median eminence in the rat. J Comp Neurol. 192: 1-19.

26. Paxinos G. Watson C. (1986). The rat brain in stereotaxic coordinates, 2nd Ed. Academic Press, London.

27. König JFR, Klippel RA. (1963). The rat brain. A stereotaxic atlas of the forebrain and lower parts of the brain stem. Williams and Wilkins, Baltimore.

28. Sawchenko PE, Swanson LW, Vale W. (1984). Co-expression of corticotropin-releasing factor and vasopressin immunoreactivity in parvicellular neurosecretory neurons of the adrenalectomized rat. Proc Natl Acad Sei USA. 81: 1883-1887. 